\title{
Collaborative and Specialized Functions of Robo1 and Robo2 in Spinal Commissural Axon Guidance
}

\author{
Alexander Jaworski, Hua Long, and Marc Tessier-Lavigne \\ Division of Research, Genentech Inc., South San Francisco, California 94080
}

Commissural neurons project axons across the floor plate at the spinal cord ventral midline. After crossing, commissural axons turn rostrally, sort into distinct positions within the ventrolateral funiculus, and never reenter the floor plate. Robo1 and Robo2 are receptors for the midline repellents Slit1-Slit3, and upregulation of Robos in post-crossing axons allows expulsion from the floor plate and prevents recrossing. Before crossing, Robo-mediated repulsion is attenuated by the divergent family member Robo3/Rig-1. To define the relative contributions of Robo family members to commissural axon guidance in mice, we studied commissural axon trajectories in combination mutants between Robo1, Robo2, and Robo3. Our results suggest the existence of another receptor contributing to Slit repulsion because the failure of midline crossing in Robo3 mutants is rescued largely but not entirely by loss of both Robo1 and Robo2 and because axon guidance defects in mice lacking both Robo1 and Robo2 are less severe than in mice lacking all Slits. Analysis of post-crossing axon trajectories indicates that Robo1 and Robo2 collaborate to prevent axons from reentering the gray matter and projecting dorsally alongside contralateral pre-crossing axons. We also discovered a previously unappreciated division of labor between Robo1 and Robo2 in post-crossing axons. Robo2 is required for axons to project away from the floor plate into the lateral funiculus. In contrast, Robo1 prevents axonal stalling after crossing. Our results reveal specialized and complementary actions of Robo1 and Robo2 in commissural axon guidance and suggest the existence of an as yet unidentified Slit receptor.

\section{Introduction}

Interneurons in the vertebrate spinal cord can be subdivided into two broad categories based on their axonal trajectories relative to the midline of the CNS. The axons of association neurons never cross the midline and instead form ipsilateral projections. The axons of commissural neurons project across the ventral midline exactly once. After crossing, commissural axons sort into specific mediolateral positions of the ventrolateral funiculus (VLF) and grow rostrally. Midline axon guidance is primarily orchestrated by the floor plate, which expresses both attractive and repulsive cues (Colamarino and Tessier-Lavigne, 1995; Tessier-Lavigne and Goodman, 1996; Kaprielian et al., 2001).

Before midline crossing, commissural axons are attracted by floor plate-derived Netrin-1 and Sonic hedgehog (Kennedy et al., 1994; Charron et al., 2003). After crossing, repellents of the Slit and Semaphorin families expel axons from the floor plate and prevent recrossing (Zou et al., 2000; Long et al., 2004). These two mechanisms operate sequentially, as precrossing axons are insensitive to midline repellents and attraction to floor plate attractants is silenced in post-crossing axons (Shirasaki et al., 1998; Zou et al., 2000; Stein and TessierLavigne, 2001).

\footnotetext{
Received Dec. 19, 2009; revised June 1, 2010; accepted June 3, 2010

This work was supported by Genentech. We thank Nona Valerde for expert technical assistance and Patrick Haddick and Jim Wong for thoughtful comments on this manuscript.

Correspondence should be addressed to Marc Tessier-Lavigne, Genentech Inc., 1 DNA Way, MS212, South San Francisco, CA 94080. E-mail: tessier-lavigne.marc@gene.com.

H. Long's present address: Rinat Laboratories, Pfizer Inc., 230 East Grand Avenue, South San Francisco, CA 94080 DOI:10.1523/JNEUROSCI.6290-09.2010

Copyright $\odot 2010$ the authors $\quad 0270-6474 / 10 / 309445-09 \$ 15.00 / 0$
}

There are three mammalian Slit homologs (Slit1-Slit3). Slits act through receptors of the Robo family, which comprises four members (Robo1-Robo4) in mammals. The three Slit genes are expressed in the floor plate and motor column of the developing spinal cord, whereas Robol and Robo2 are broadly expressed in the spinal cord, including commissural neurons (Itoh et al., 1998; Brose et al., 1999; Li et al., 1999). Robo1 and Robo2 protein expression is low on axons of association and commissural neurons that are navigating the gray matter and is increased when these axons enter the ipsilateral or contralateral VLF, respectively (Long et al., 2004). Robo3, which is expressed exclusively in commissural neurons, produces two splice isoforms with opposite activities (Sabatier et al., 2004; Chen et al., 2008). Robo3.1 is expressed on pre-crossing axons and suppresses premature responsiveness to Slits, thereby allowing midline crossing; Robo3.2 localizes to post-crossing axons and acts as a classical Slit receptor that contributes to midline repulsion (Chen et al., 2008).

We have studied the detailed contributions of the three neuronally expressed Robos by analyzing commissural axon behavior in mice lacking all possible combinations of Robo1, Robo2, and Robo3. We find that Robol and Robo2 are responsible for some but not all Slit-mediated expulsion of commissural axons from the floor plate, suggesting the existence of another Slit receptor. This possibility is further supported by our finding that loss of Robo1 and Robo2 does not fully rescue midline crossing in mice lacking Robo3, although this result could alternatively support a role for Robo3 as a receptor for a putative midline attractant. Robo 1 and Robo 2 are also required to prevent post-crossing axons from leaving the VLF and entering the gray matter. Finally, Robo1 and Robo2 fulfill separate, specialized functions in post- 
crossing axons, because Robo 2 is required for axons to project away from the floor plate into the lateral funiculus (LF), whereas Robol prevents axons from stalling after crossing.

\section{Materials and Methods}

Mouse strains. Null alleles for Slit1, Slit2, and Slit3 have been described previously, and mice carrying these mutations were genotyped as originally reported (Plump et al., 2002; Yuan et al., 2003). Robo1, Robo2, and Robo3 null alleles have been described previously, and genotyping was performed as described previously (Grieshammer et al., 2004; Long et al., 2004; Sabatier et al., 2004). The generation of Robol ${ }^{-1-} ; R_{0 b o 2^{-\prime-}}$ mice has been reported previously (Chen et al., 2008). Mice were maintained on a CD-1 background, except for the Robo1 mutants and Robo1/3 mutants, which were on a C57BL/6 or mixed (C57BL/6 and CD-1) background, respectively. The size of the spinal cord and the thickness of the ventral funiculus (VF) were not statistically different between embryonic day 11.5 (E11.5) embryos from the different genetic backgrounds used (data not shown), indicating that these embryos are comparable. All analyses were performed without distinguishing between male and female embryos.

Immunohistochemistry. Unless indicated otherwise, all incubations were performed at room temperature. Embryos were fixed overnight at $4^{\circ} \mathrm{C}$ in PBS containing 4\% paraformaldehyde (PFA), washed three times in PBS, incubated in $30 \%$ sucrose in PBS at $4^{\circ} \mathrm{C}$ overnight, and embedded in OCT. Transverse cryostat sections $(20 \mu \mathrm{m})$ were blocked for $1 \mathrm{~h}$ in $2.5 \%$ heat-inactivated goat serum and $0.1 \%$ Triton X-100 in PBS and incubated with primary antibodies diluted in blocking solution at $4^{\circ} \mathrm{C}$ overnight. After three washes (10 min each) in blocking solution, sections were incubated with secondary antibodies in blocking solution for $2 \mathrm{~h}$, washed three times for $10 \mathrm{~min}$ in blocking solution, and mounted using Fluoromount G (Electron Microscopy Sciences). Images were acquired on a Zeiss Axioplan 2.

Concentrated mouse monoclonal antibodies against TAG-1 (clone 4D7; 1:200) and neurofilament (clone 2H3; 1:1000) were obtained from the Developmental Studies Hybridoma Bank (University of Iowa, Iowa City, IA). The rat monoclonal antibody against L1 (1:200) was from Millipore Bioscience Research Reagents. Rabbit polyclonal antibodies against green fluorescent protein (GFP) (1:400) and mouse monoclonal antibody against $\beta$-galactosidase ( $\beta$-Gal) $(1: 1000)$ were from Invitrogen. The rabbit polyclonal antibody against the Robo3 extracellular domain has been described previously (Yuan et al., 1999). Secondary antibodies were Alexa488-conjugated goat anti-mouse (1:200), Alexa488conjugated goat anti-rat (1:200), Alexa594-conjugated goat anti-rabbit (1:200), and Alexa594-conjugated goat anti-mouse IgM (1:200), all from Invitrogen.

Quantification of commissure, VF, and LF thickness. Four forelimb level spinal cord sections per embryo were used for quantifications. NIH Image was used to quantify the thickness of the commissure, the VF, and the LF in L1 immunostained sections. The VF and LF thickness was measured on both sides of the spinal cord at the levels indicated by arrowheads in Figures 3 and 4. The values were normalized to the distance between roof plate and floor plate for each section. Mean values from multiple mice ( $n$ is indicated in the figures) with the same genotype were determined and analyzed for statistically significant differences by one-way ANOVA. Post hoc analysis of wild-type, Robo3, Robo1/3, Robo2/3, and Robo1/2/3 mutant commissure thickness was done using Holm's test for multiple comparisons with $\alpha=0.1$. Post hoc comparison of VF or LF thickness in Robo1, Robo2, and Robol/2 mutants against wild-type and Robo1/3, Robo2/3, and Robo1/2/3 mutants against Robo3 mutants was done using Holm's test with $\alpha=0.05$. Values for $\alpha$ were chosen to keep the most stringent adjusted $\alpha \geq 0.01$.

Quantification of L1 immunoreactivity. Four forelimb level spinal cord sections per embryo were used for quantification using NIH ImageJ. We measured pre-crossing and post-crossing L1 staining in line scans. Background fluorescence measured in the ventricular zone was subtracted from these values, and pre-crossing intensity was expressed as a fraction of post-crossing L1 staining. Post-crossing L1 immunoreactivity did not differ between genotypes (data not shown). Mean values from multiple mice per genotype (same $n$ as used for VF and LF measurements) were determined and compared with wild-type in a two-tailed $t$ test.

DiI tracing. For tracing of commissural axons in the open-book configuration, the roof plate was removed from dissected spinal cords, and preparations were embedded in collagen. After overnight fixation in $4 \%$ PFA at $4^{\circ} \mathrm{C}$ and three 10 min washes in PBS, the lipophilic dye CM-DiI (Invitrogen) was injected dorsally into one side of the spinal cord. Injection was performed at several anteroposterior positions in each preparation. After $24-30 \mathrm{~h}$, the open-book preparations were mounted in Fluoromount $\mathrm{G}$ for immediate image acquisition using a Zeiss Axioplan 2.

For tracing of commissural axons in transverse sections, embryos were fixed overnight at $4^{\circ} \mathrm{C}$ in PBS containing 4\% PFA. After three $10 \mathrm{~min}$ washes in PBS, CM-DiI was injected dorsally into one side of the spinal cord. Each embryo was injected at several anteroposterior positions. Twenty-four hours later, embryos were embedded in 5\% agarose in PBS, and vibratome sections $(100 \mu \mathrm{m})$ were mounted in Fluoromount $\mathrm{G}$ and immediately examined on a Zeiss Axioplan 2.

Quantification of DiI tracing. For injections in open-book preparations, each injection site was scored as explained in the figure legends. For DiI tracing in transverse sections, each section was scored as displaying the phenotype shown in Figure 5 or as normal. $n$ is indicated in the figures and figure legends.

In situ hybridization. In situ hybridization on mouse spinal cord sections was performed as described previously (Sabatier et al., 2004).

\section{Results}

\section{Robo1 and Robo2 mediate some but not all aspects of Slit function}

We previously documented a variety of commissural axon guidance defects in mice lacking all three Slits and mice lacking either Robo1 or Robo2 (Long et al., 2004). In Slit1/2/3 mutants, we reported that axons are abnormally defasciculated as they approach and cross the midline, that there is an increased number of growth cones in the floor plate and midline recrossing of axons, and that post-crossing axons appear shifted from the LF to the VF. Although the midline guidance defects are partially phenocopied in Robol mutants and the post-crossing defects are partially phenocopied in Robo2 mutants, it remained unclear whether the combined actions of Robol and Robo2 could account for all Slit signaling in commissural axon guidance. We therefore analyzed commissural axon guidance before and during midline crossing in Robol/2 double-mutant mice [which had to be generated by chromosomal recombination, because the two genes are closely linked on chromosome 16 (Chen et al., 2008)] (Fig. 1). First, we performed TAG-1 immunostaining of E11.5 brachial spinal cord sections. In wild-type embryos, TAG-1 labels commissural axons as they approach and cross the midline, and some TAG-1 staining is seen in the most medial part of the VF (Fig. 1A). TAG-1 also labels sensory axons, and, as reported previously (Ma and Tessier-Lavigne, 2007), sensory axons overshoot the dorsal root entry zone in Robo1/2 mutant embryos (Fig. $1 B$ ) but not wild-type, a phenotype also seen in Slit2 mutants (Ma and Tessier-Lavigne, 2007). However, whereas in the Slit1/2/3 mutant, pre-crossing axons defasciculate and TAG-1 is absent from the medial VF (Long et al., 2004), TAG-1-positive commissural axons in the Robo1/2 mutant project toward the floor plate in a pattern indistinguishable from wild-type, and TAG-1 immunoreactivity is present in the medial VF (Fig. $1 B$ ). Thus, loss of both Robo1 and Robo2 does not reproduce all commissural axon guidance defects observed in Slit1/2/3 mutants.

To analyze axon behavior during midline crossing in detail, we labeled commissural axons by DiI injection into the dorsal part of E12.5 spinal cord open-book preparations (Fig. 1C-E). Whereas axonal growth cones are rarely (18\% of injection sites) 

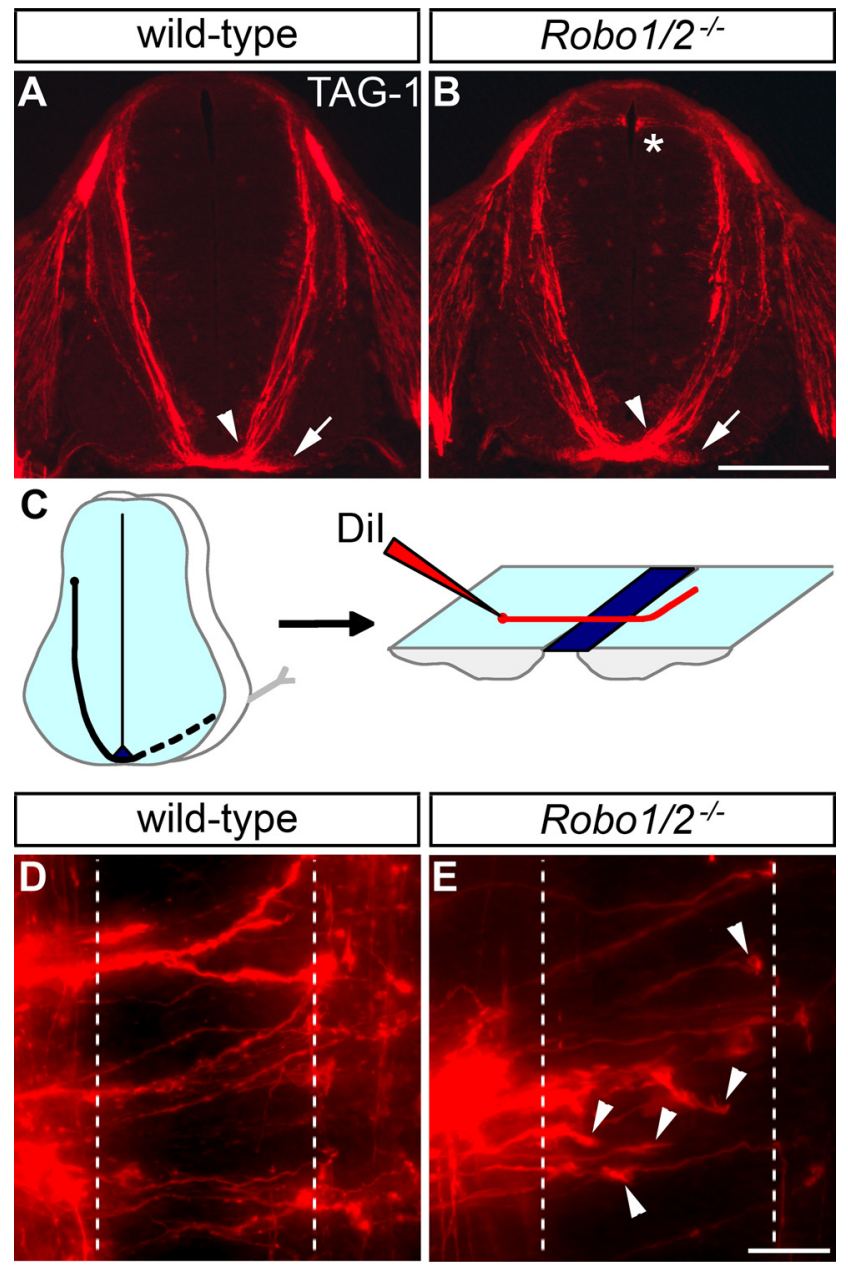

Figure 1. Commissural axon guidance in mice lacking Robo1 and Robo2 is affected less severely than in Slit1/2/3 mutants. $\boldsymbol{A}, \boldsymbol{B}$, Transverse sections of $\mathrm{E} 11.5$ spinal cords from wildtype and Robo1 $1^{-1-} ;$ Robo2 $2^{-1-}$ mice were stained for TAG-1 to label commissural axons as they grow ventrally toward the floor plate and cross to the contralateral side. Commissural axons are tightly fasciculated as they approach and cross the midline (arrowhead) in both wild-type ( $\boldsymbol{A}$ ) and Robo1/2 mutant ( $\boldsymbol{B}$ ) embryos. TAG-1 staining in the medial VF (arrow) is also observed in both genotypes. TAG-1-positive sensory afferents misproject into the dorsal spina cord of Rob01/2 mutants $\left(^{*}\right)$, which is not seen in wild-type. C, A schematic drawing of commissural axon projection toward and across the floor plate, as visualized by Dil injection in an open-book preparation. $\boldsymbol{D}, \boldsymbol{E}$, Open books of E12.5 wild-type and $R o b 01^{-1-} ; R o b 02^{-1-} \mathrm{em}$ bryos were injected with Dil as shown in $\boldsymbol{C}$. In wild-type mice $(\boldsymbol{D})$, axons project across the ipsilateral (left dashed line) and contralateral (right dashed line) floor plate borders and then turn anteriorly (up). In mice lacking Robo1 and Robo2 (E), many commissural axon growth cones are observed in the floor plate (arrowheads). Injection sites with five or more growth cones in the floor plate are more frequent in Robo1/2 mutants ( $45 \%$ of 42 injections in 5 embryos) than in wild type ( $18 \%$ of 40 injections in 5 embryos). Scale bars: $\boldsymbol{A}, \boldsymbol{B}, 200 \mu \mathrm{m}$; D, E, $25 \mu \mathrm{m}$.

observed in the floor plate of wild-type embryos (Fig. $1 D$ ), they are frequently (45\%) seen in mice lacking Robo1 and Robo2 (Fig. $1 E)$, suggesting axonal stalling. This phenotype is less prominent in Robo1/2 mutants than in Slit1/2/3 mutants (73\%) (Long et al., 2004). Thus, loss of all Slits causes more severe commissural axons guidance defects than loss of both Robol and Robo2.

Robo3 allows midline crossing by suppressing

Robo1/2-mediated midline repulsion and through additional mechanisms

Robo3 suppresses Slit responsiveness in pre-crossing commissural axons, and midline crossing in Robo3 mutant mice can be partially rescued by loss of Robo1 or both Slit1 and Slit2 (Sabatier et al., 2004). We generated combination mutants between Robo1, Robo2, and Robo3 to assess the contributions of Robol and Robo2 to premature midline repulsion in Robo3 mutants (Fig. 2). Axons were labeled by neurofilament staining in E11.5 brachial spinal cord transverse sections, and the amount of midline crossing in the different mutants was quantified (see Materials and Methods). In wild-type embryos, a tight fascicle of axons forms under the floor plate (Fig. 2A). In Robo1 and Robo1/2 mutant embryos, we frequently observed axons that projected dorsally from the ventral commissure toward the ventricular zone (Fig. $2 C, G)$. However, the thickness of the main commissural bundle in mice lacking Robo1, Robo 2 or both Robo1 and Robo2 is similar to wild type (normalized commissure thickness: wild-type, $0.0793 \pm 0.0034 ; R_{\text {Robol }}^{-/-}, 0.0724 \pm 0.0057 ;$ Robo2 $^{-/-}$, $0.0855 \pm 0.0061 ;$ Robol $^{-/-} ;$Robo2 $2^{-/-}, 0.0864 \pm 0.0092$ ) (Fig. $2 C, E, G, I)$. As reported previously (Sabatier et al., 2004), the ventral commissure is absent in Robo3 mutant embryos (commissure thickness, $0.0005 \pm 0.0005 ; p<0.0001$ compared with wild type), and midline crossing is partially but consistently rescued in Robo1/3 double mutants (commissure thickness, $0.0156 \pm$ 0.0006; $p=0.009$ compared with Robo3 ${ }^{-1-} ; p<0.0001$ compared with wild type) (Fig. $2 B, D$ ). In a few cases, axons were observed in the ventral commissure of embryos lacking both Robo2 and Robo3, but the amount of rescue is not statistically significant compared with Robo3 mutants (commissure thickness, $0.0023 \pm 0.0018$ ) (Fig. $2 F$ ). However, we found that loss of Robo1 and Robo2 in a Robo3 mutant background restores significantly more midline crossing than loss of Robol alone (commissure thickness, $0.0346 \pm 0.0048 ; p=0.0069$ ) (Fig. $2 H$ ). These results suggest that failure to cross the floor plate in Robo3 mutant mice is caused by Robol-dependent midline repulsion in some axons and Robol- and Robo2-mediated repulsion in other axons.

The finding of a role for Robo 2 was surprising because previous results suggested that Robo 2 and Robo 3 mRNAs are expressed in nonoverlapping neuronal populations in the developing spinal cord (Sabatier et al., 2004). However, we found that Robo3 protein is expressed in a broader population of commissural neurons than those marked by high Robo3 mRNA expression (supplemental Fig. S1 A, B, available at www.jneurosci.org as supplemental material). This disparity between mRNA and protein expression was also observed when we compared the expression of GFP protein and GFP mRNA expressed from the Robo3 locus in Robo $3^{+/-}$ embryos (supplemental Fig. S1C,D, available at www.jneurosci. org as supplemental material). A likely interpretation of these results is that neurons that express Robo3 mRNA will later switch off the transcript, but the protein perdures in the cells; alternatively, the cells expressing Robo3 protein may express lower levels of Robo3 mRNA not detected by our in situ hybridization protocol. Whatever the explanation, it seems that Robo3 protein is expressed more broadly than its mRNA. To determine whether these Robo3-expressing neurons also express Robo2, we examined GFP expression from the Robo 3 locus and $\beta$-Gal expression from the Robo2 locus in the same animal (supplemental Fig. S2, available at www.jneurosci.org as supplemental material). We performed GFP and $\beta$-Gal immunohistochemistry on E11.5 brachial spinal cord transverse sections from $\mathrm{Robo2}^{+/-}$; $R o b o 3^{+/-}$embryos and found that a subset of commissural axons expresses both GFP and $\beta$-Gal (supplemental Fig. S2 $B, D$, available at www.jneurosci.org as supplemental material). These results are consistent with the idea that some neurons coexpress 


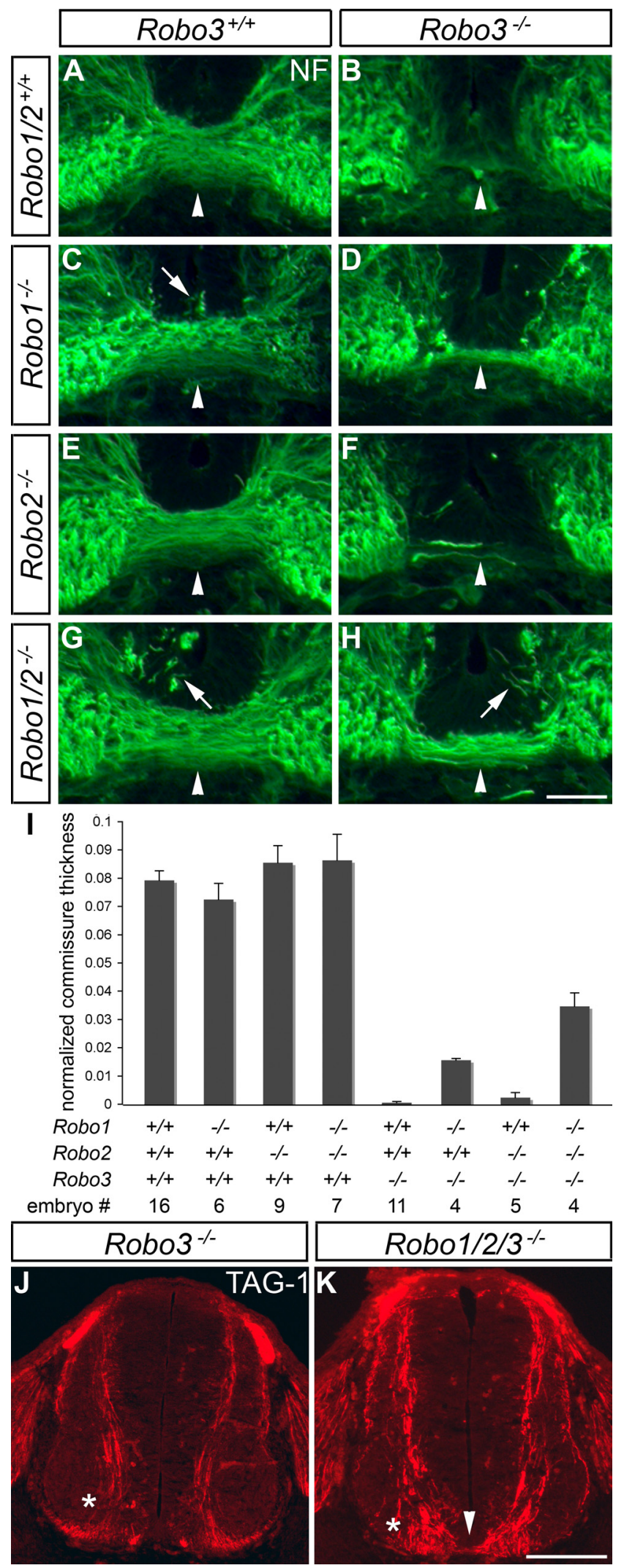

Figure 2. L L L A-I, Transverse sections of E11.5 spinal cords from mice with the indicated genotypes were stained for neurofilament (NF), and the thickness of the main commissural bundle was quantified (see Materials and Methods). In wild-type embryos $(\boldsymbol{A})$, axons form a compact fascicle (arrowhead) under the floor plate. In Robo3 mutant mice $(\boldsymbol{B})$, axons fail to cross the midline. In mice lacking Robo1 (C), some axons invade the floor plate (arrow), but the main commissural
Robo2 and Robo3, helping explain why additional loss of Robo2 affects the phenotype of Robo1/3 mutant animals.

Unexpectedly, the amount of midline crossing in Robol/2/3 mutants is still significantly lower than in wild-type embryos $(p<0.0001)$. This incomplete rescue of commissure formation in Robo1/2/3 mutants suggests that many commissural axons remain ipsilaterally. To address this possibility, we stained E11.5 brachial spinal cord transverse sections for TAG-1 to specifically label pre-crossing commissural axons. As they grow ventrally, all TAG-1-positive axons in Robo3 mutants veer away from the floor plate and enter the ipsilateral VLF (Fig. $2 J$ ). In Robo1/2/3 mutants, some axons stay ipsilaterally, whereas others approach and cross the midline (Fig. $2 \mathrm{~K}$ ). These results indicate that a yet to be defined Robo3-dependent mechanism not involving repression of Robo1/2 signaling is required to prevent ipsilateral projection and allow midline crossing in a subset of commissural axons.

Robo1 and Robo 2 control distinct aspects of post-crossing commissural axon guidance in the ventrolateral funiculus We next focused on the functions of Robol and Robo 2 in commissural axons after midline crossing and analyzed post-crossing axon behavior in mice lacking Robo1, Robo2, or both Robo1 and Robo2 (Fig. 3). We stained E11.5 brachial spinal cord transverse sections for L1 to label axons in the VLF and quantified the thickness of the ventral and lateral funiculi. In mice lacking Robo1, the VF is thinner than in wild type (normalized thickness: wild type, $0.1009 \pm 0.0035 ;$ Robo1 $\left.^{-/-}, 0.0805 \pm 0.007 ; p=0.0322\right)$, but the LF appears normal (thickness: wild type, $0.0352 \pm 0.002$; Robo1 ${ }^{-1-}, 0.0337 \pm 0.0043$ ) (Fig. $3 A, B$ ). Conversely, the VF is expanded in Robo2 mutants (thickness, $0.1265 \pm 0.0079 ; p=$ 0.0027), whereas the LF is thinner than in wild type (thickness, $0.0256 \pm 0.0022 ; p=0.006$ ) (Fig. 3C). In Robol/2 mutant embryos, the thickness of the VF is similar to wild type $(0.1036 \pm$ 0.0091), whereas the LF is dramatically thinned (thickness, $0.0172 \pm 0.0021 ; p<0.0001$ ) (Fig. 3D). These results suggest that Robo1 and Robo2 regulate sorting of axons into different parts of the VLF.

The changes in axonal distribution between the VF and LF in the different Robol/2 single and double mutants could reflect changes in ipsilaterally projecting axons, post-crossing commissural axons, or both. To specifically assess the functions of Robo 1 and Robo 2 in post-crossing commissural axons, we performed anterograde labeling by DiI injection into the dorsal part of E11.5 spinal cord open-book preparations and analyzed the trajectories of axons after floor plate crossing. In the majority (88\%) of injections performed in wild-type embryos, post-crossing axons turn rostrally, project along the contralateral side of the floor plate for

fascicle is comparable in size with wild-type embryos. Loss of Robo1 in a Robo3 mutant background (D) partially rescues midline crossing ( $p=0.009$ when compared with Robo3 ${ }^{-1-}$; $p<0.0001$ when compared with wild type). The ventral commissure in $R_{0 b 02^{-1-}}$ mice $(\boldsymbol{E})$ looks similar to wild-type mice, and loss of Robo2 in a Robo3 ${ }^{-/-}$background $(\boldsymbol{F})$ restores midline crossing in a few cases (not statistically significant) but less than observed in $R_{0 b 01^{-1-}} ; R_{0 b 03^{-1-}}$ embryos. Axons are invading the floor plate (arrow) in mice lacking Robo1 and Robo2 (G), but the commissure is similar in size to wild-type embryos. In Robo ${ }^{-1-}$; Robo2 $2^{-/-} ; R_{0 b 03^{-1-}}$ mice $(\boldsymbol{H})$, the commissure is thicker than in mice lacking Robo1 and Robo3 ( $p=0.0069)$ but is still significantly thinner than in wild-type embryos $(p<0.0001)$. Error bars in I indicate SEM. J, $\boldsymbol{K}$, TAG-1 staining of E11.5 spinal cord sections labels commissural axons as they approach and cross the midline. In Robo3 mutants $(J)$, all commissural axons stay

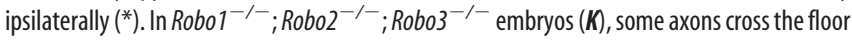
plate (arrowhead), whereas others veer away from the midline and stay on the ipsilateral side $\left.{ }^{*}\right)$. Scale bars: $\boldsymbol{A}-\boldsymbol{H}, 50 \mu \mathrm{m} ; \boldsymbol{J}, \boldsymbol{K}, 200 \mu \mathrm{m}$. 


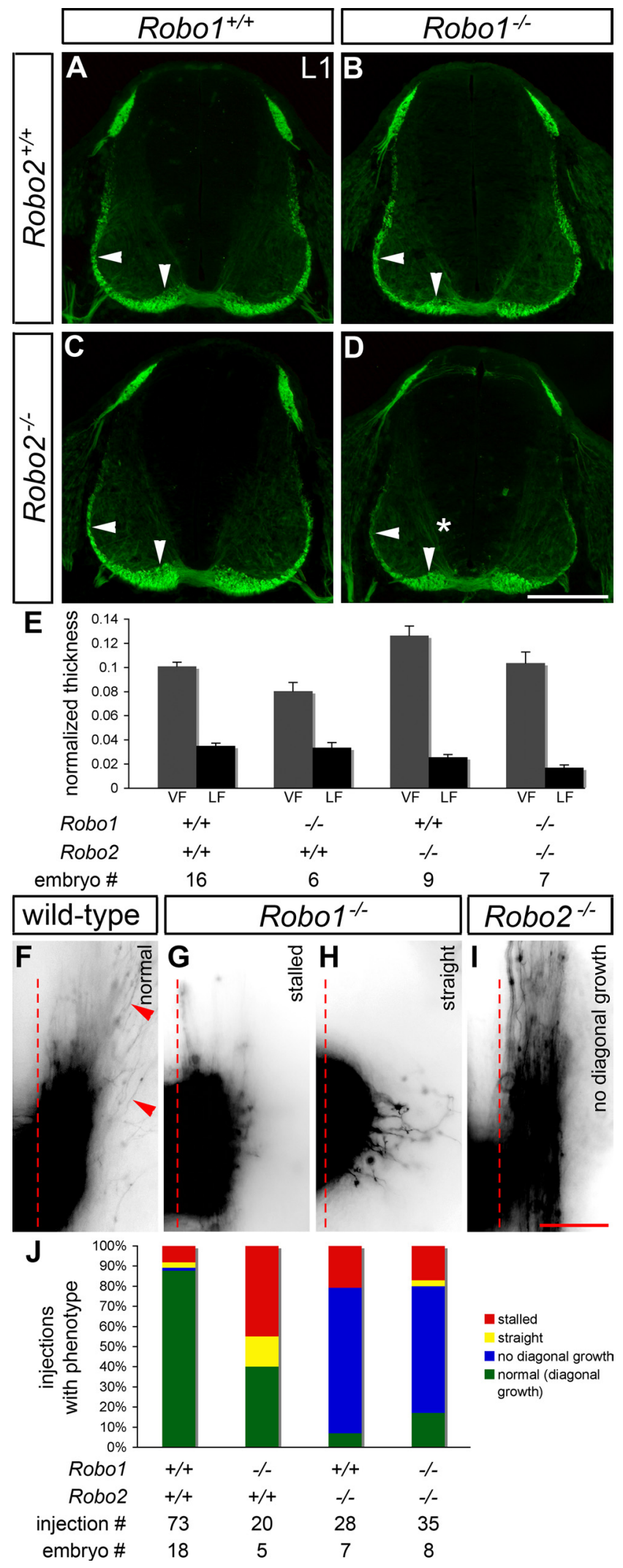

Figure 3. Sorting of post-crossing commissural axons into the ventral and lateral funiculi is abnormal in mice lacking Robo1, Robo2, or Robo1 and Robo2. A-E, Transverse sections of E11.5 spinal cords from mice with the indicated genotypes were stained for $\mathrm{L} 1$ to label post-crossing commissural axons, and the thickness of the VF and LF was quantified (see Materials and Methods). In Robo1 mutant embryos (B), the VF (vertical arrowhead) is thinner than in wild-type embryos (A) ( $p=0.0322)$. In mice lacking Robo2 ( $)$, the VF is thicker than in wild type ( $p=$ varying distances, and then leave the vicinity of the floor plate, growing dorsally and rostrally (Fig. $3 F, J$ ). These diagonally growing axons can extend well into the contralateral LF (supplemental Fig. S3, available at www.jneurosci.org as supplemental material). This projection pattern has been described previously for a large (90\%) subset of commissural axons (Imondi and Kaprielian, 2001). In contrast, in Robol mutant embryos, only $40 \%$ of the injection sites exhibit the same phenotype, whereas most axons in $45 \%$ of the injection sites appear to stall just after leaving the floor plate (Fig. $3 G$ ). This phenotype is seen in only $8 \%$ of injections in wild-type spinal cords. An exclusively straight projection pattern without rostral turn was also seen in these Robol mutants (15\%) (Fig. 3H). This is rarely seen in wild-type embryos (3\%), but straight projections are sometimes seen intermixed with the predominant diagonal pattern (data not shown). In both Robo2 mutants and mice lacking Robo1 and Robo2, the predominant phenotype ( 72 and $63 \%$, respectively) is a failure of post-crossing axons to leave the vicinity of the floor plate while projecting rostrally (Fig. $3 I$ and data not shown). This is never observed in Robol mutant embryos and only rarely (1\%) in wild type. Stalling is also increased in $R_{o b o 2^{-/}}$and Robo1 ${ }^{-/-}$; Robo $2^{-/-}$mice (21 and $17 \%$, respectively), whereas exclusively straight growth of commissural axons is not observed in Robo2 mutants and rarely (3\%) in Robo1/2 mutants. These results indicate that Robol, in addition to preventing stalling during floor plate crossing, allows commissural axons to grow after they leave the floor plate. Robo2, conversely, is required for post-crossing commissural axons to project away from the midline into the LF. Moreover, and perhaps surprisingly, the rescue of stalling after crossing in mice lacking both Robos suggests that stalling in Robo1 mutant mice is caused by Robo2.

Robo-dependent sorting of axons into the ventral and lateral funiculi does not require midline crossing

Guidance of axons into distinct parts of the VLF by Robol and Robo2 might require midline crossing or might instead also occur in ipsilaterally projecting axons, as well. To distinguish these possibilities, we analyzed axonal projections in combination mutants of Robo1 and Robo2 with Robo3, in which most or all commissural axons stay ipsilaterally because of the lack of Robo3 (Fig. 4). L1 immunostaining of E11.5 brachial spinal cord transverse sections labels axons in the VLF. In Robo3 ${ }^{-/-}$embryos, both the VF and the LF are comparable in size with wild type (VF thickness, $0.0956 \pm 0.0054$; LF thickness, $0.0349 \pm 0.0032$ ) (compare Figs. $4 A, E, 3 A, E$ ). In mice lacking Robo1 and Robo3, the VF is

0.0027), whereas the LF (horizontal arrowhead) is thinned ( $p=0.006)$. Thinning of the LF is also observed in Robo $1^{-/-} ;$Robo2 $^{-/-}$embryos $(\boldsymbol{D})(p<0.0001)$, but the VF appears similar to wild type. L1 immunostaining is observed in association with pre-crossing segments of commissural axons (*) in embryos lacking Robo1 and Robo2 (D). Error bars in $\boldsymbol{E}$ indicate SEM.

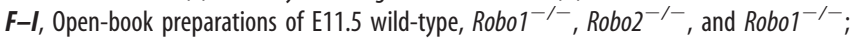
Robo2 ${ }^{-/-}$embryos were injected with Dil as described in Figure 1. The examples shown are inverted grayscale images. In wild-type embryos $(\boldsymbol{F})$, commissural axons turn anteriorly (top) after crossing the contralateral border of the floor plate (dashed line). Many axons (arrowheads) project diagonally away from the floor plate, as they start growing dorsally while continuing anteriorly. In Robo1 mutant embryos $(\boldsymbol{G}, \boldsymbol{H})$, axons often stall just after leaving the floor plate (G) or fail to turn longitudinally and instead grow dorsally $(\boldsymbol{H})$. In Robo2 ${ }^{-/-}(\boldsymbol{I})$ and $R o b 01^{-1-}$; Robo2 $2^{-1-}$ (data not shown) embryos, axons turn anteriorly after crossing but fail to grow dorsally.J, A quantification of the Dil analysis. Stalling, as shown in $\mathbf{G}$, and straight growth after crossing (see $\boldsymbol{H}$ ) are common in Robo 1 mutants, whereas a failure to project diagonally (see $\boldsymbol{I}$ ) is observed in the majority of injection sites for Robo2 and Robo 1/2 mutants. Scale bars: $A-D, 200$ $\mu \mathrm{m} ; \boldsymbol{F}-\boldsymbol{I}, 50 \mu \mathrm{m}$ 


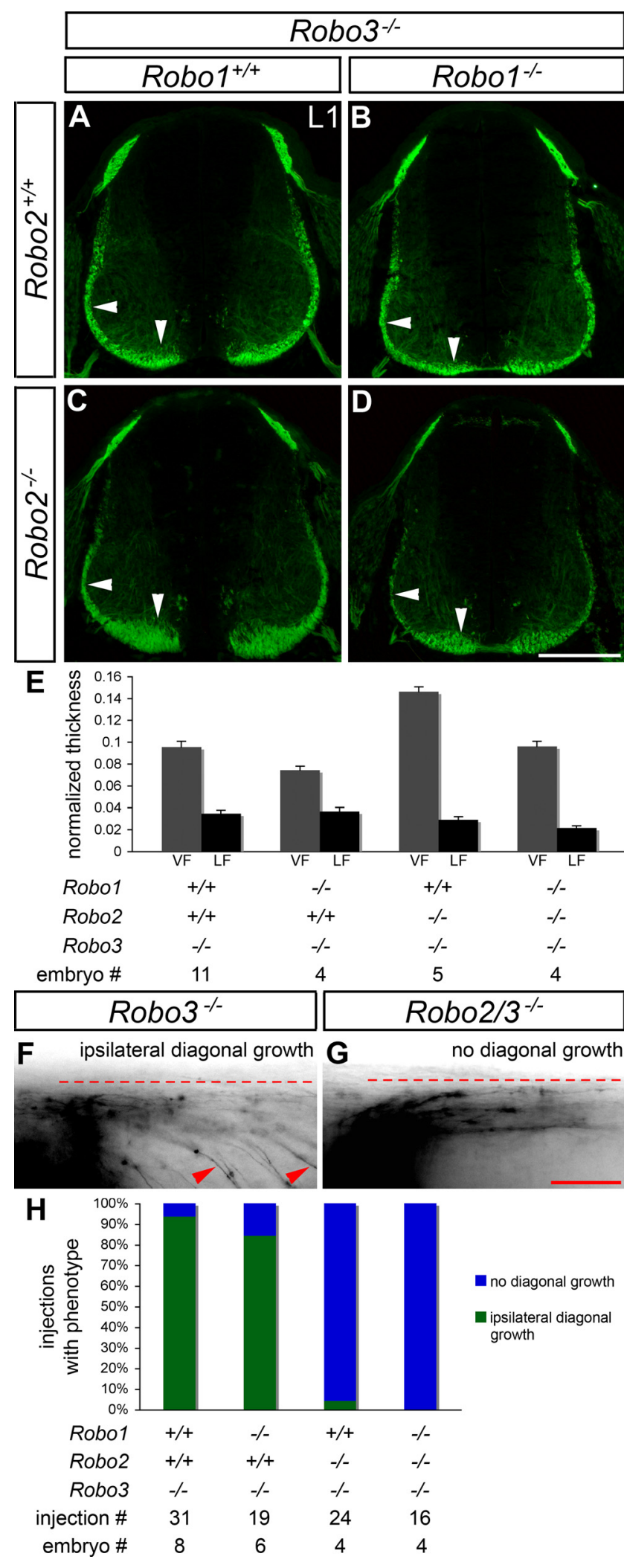

Figure 4. Projection of ipsilateral axons into the ventral and lateral funiculi of Robo3 mutant mice is altered by lack of Robo1, Robo2, or Robo1 and Robo2. A-E, Transverse sections of E11.5 spinal cords from mice with the indicated genotypes were stained for L1, and the thickness of the VF and the LF was quantified (see Materials and Methods). In Robo3 mutant embryos (A), formation of the VF (vertical arrowhead) and LF (horizontal arrowhead) appears similar to wild-type embryos (compare with Fig. $3 A$ ). In mice lacking Robol and Robo3 (B), the VF is thinner than in Robo3 ${ }^{-1-}$ embryos $(p=0.0184)$. Loss of Robo2 in a Robo3 mutant background $(\boldsymbol{C})$ leads to an expansion of the VF ( $p<0.0001)$, whereas the LF appears slightly thinner than in Robo3 mutants (thickness, $0.0745 \pm 0.0036 ; p=$ 0.0184 ), whereas the LF is normal in size (thickness, $0.0367 \pm$ 0.0039) (Fig. 4B). In Robo2 ${ }^{-/-}$; Robo ${ }^{-/-}$embryos, the VF is thicker than in Robo3 mutants (thickness, $0.1464 \pm 0.0045$; $p<$ 0.0001 ), and the LF is slightly thinned, but this change is not statistically significant (thickness, $0.0292 \pm 0.0029$ ) (Fig. 4C). In mice lacking Robo1, Robo2, and Robo3, the VF is similar in size to Robo3 mutants (thickness, $0.0961 \pm 0.0047$ ), whereas the LF is significantly smaller (thickness, $0.0219 \pm 0.0018 ; p=0.0172$ ). Thus, Robo1 and Robo2 regulate sorting of axons into different portions of the ipsilateral VLF in a Robo3 mutant background. Because the observed defects are very similar to the defects in contralateral axonal sorting in mice lacking Robo1, Robo2, or both Robo1 and Robo2 in a Robo $3^{+/+}$background, these results suggest that the same Robo1- and/or Robo2-mediated mechanisms guide both ipsilaterally and contralaterally projecting axons into different parts of the VLF. These data also indicate that neither Robo3.1 nor Robo3.2 (which are both absent in the Robo3 mutant) make a significant contribution to axonal sorting in the VLF.

To investigate ipsilateral sorting by Robol and Robo2 more directly, we performed DiI injections into the dorsal part of E11.5 spinal cord open-book preparations and analyzed the trajectories of axons on the ipsilateral side of the floor plate. Perhaps surprisingly, we found that, in the majority of injections performed in Robo3 $^{-1-}$ (94\%) and Robo1 ${ }^{-1-}$; Robo3 ${ }^{-1-}$ embryos (84\%), axons turn rostrally at the ipsilateral border of the floor plate, project along the midline for varying distances, and then leave the vicinity of the floor plate growing dorsally and rostrally (Fig. $4 F, H$, and data not shown). In contrast, axons fail to leave the immediate vicinity of the floor plate in Robo2/3 and Robol/2/3 mutants after making their ipsilateral rostral turn ( $96 \%$ of injections for Robo2/3 mutants and 100\% for Robo1/2/3 mutants) (Fig. $4 G$ and data not shown). Thus, in Robo3 mutants, Robo2 is required for ipsilateral axons to project away from the midline into the LF, similar to what is observed for contralateral axons in Robo $^{+/+}$mice.

Robo1 and Robo2 are required to prevent post-crossing axons from reentering the gray matter after midline crossing Similar to Slit1/2/3 triple mutants (Long et al., 2004), we observed increased L1 immunoreactivity associated with the ventral portion of pre-crossing commissural axons in mice lacking Robol and Robo 2 (relative pre-crossing intensity, $34.0 \pm 2.4 \%$ of postcrossing intensity; $p<0.0001)$ compared with wild type (19.3 \pm $1.1 \%)$, which is not seen in Robo2 mutants $(17.5 \pm 0.8 \%)$ and to a lesser extent in Robo1 mutants $(24.2 \pm 1.8 \% ; p=0.0359)$ (Fig. $3 A-D)$. It is unclear whether this phenotype is attributable to

\footnotetext{
$\leftarrow$

thinned (not statistically significant). In Robo $1^{-1-} ; R_{0 b 02^{-1-}} ; R_{0 b 03^{-1-}}$ embryos (D), the VF looks normal, whereas the LF is thinner than in Robo3 mutants $(p=0.0172)$. Error bars in $\boldsymbol{E}$

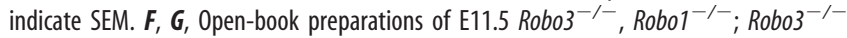
Robo2 $^{-1-} ;$ Robo3 $^{-1-}$, and Robo $1^{-1-} ;$ Robo2 $^{-1-} ;$ Robo3 $^{-1-}$ embryos were injected with Dil as described in Figure 1. The examples shown are inverted grayscale images. In $\mathrm{Robo}^{-1-}$ (F) and Robo1 ${ }^{-1-} ; R_{0 b 03^{-1-}}$ (data not shown) embryos, commissural axons grow to the ipsilateral border of the floor plate (dashed line), turn anteriorly (right), and project diagonally away from the floor plate while staying ipsilaterally (arrowheads). In Robo2/3 (G) and Robo 1/ $2 / 3$ (data not shown) mutants, ipsilaterally projecting commissural axons project anteriorly in close apposition to the floor plate without diagonal growth. $\boldsymbol{H}$, A quantification of the Dil analysis. A failure to project diagonally (see $\boldsymbol{G}$ ) is observed in the majority of injection sites for Robo2/3 and Robo1/2/3 mutants. Error bars indicate SEM. Scale bars: A-D, $200 \mu \mathrm{m}$; $\boldsymbol{F}, \mathbf{G}, 50 \mu \mathrm{m}$
} 

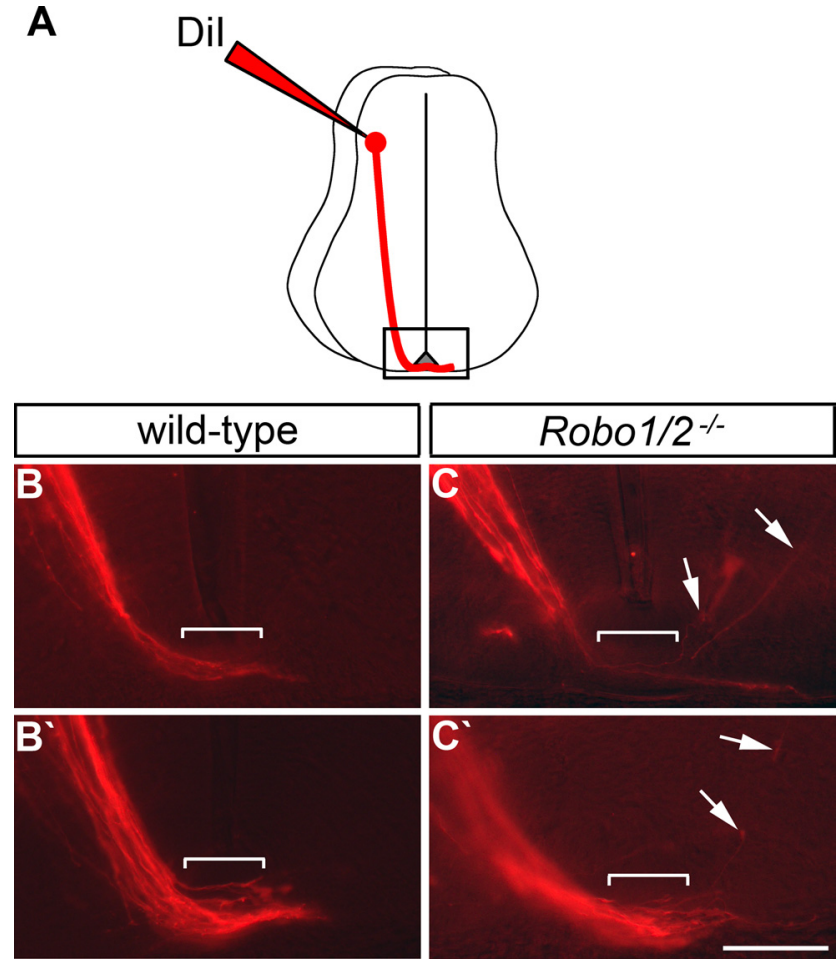

Figure 5. Post-crossing axons aberrantly enter the gray matter in mice lacking Robo1 and Robo2. A, A schematic drawing of commissural axon projection toward and across the floor plate, as visualized by Dil injection. The boxed area indicates the part of the spinal cord shown in $\boldsymbol{B}, \boldsymbol{B}^{\prime}, \boldsymbol{C}$, and $\boldsymbol{C}^{\prime}$. $\boldsymbol{B}, \boldsymbol{C}$, E11.5 spinal cords from embryos with the indicated genotypes were injected with Dil as shown in $\boldsymbol{A}$. Dil-labeled axons were then visualized in transverse sections. Two examples of wild-type spinal cords $\left(\boldsymbol{B}, \boldsymbol{B}^{\prime}\right)$ show axons crossing the floor plate (brackets) and entering the contralateral VF. In Robo1 $1^{-1-} ; R_{0 b 02^{-1-}}$ embryos $\left(\boldsymbol{C}, \boldsymbol{C}^{\prime}\right)$, most axons enter the contralateral VF, but some (arrows) enter the gray matter after midline crossing. Postcrossing axons entering the gray matter are observed more frequently in mice lacking Robo 1 and Robo2 (38\% of 24 sections from 12 embryos) than in wild-type ( $7 \%$ of 27 sections from 13 embryos). Scale bar, $100 \mu \mathrm{m}$.

elevated expression of L1 on pre-crossing axons or a result of strongly L1-positive post-crossing axons projecting in ectopic positions. To distinguish between these possibilities, we traced commissural axons by unilateral DiI injection into the dorsal part of E11.5 whole spinal cords of wild-type and Robo1/2 mutant mice and analyzed post-crossing axonal projections in transverse sections (Fig. 5). In wild-type embryos, DiI-labeled axons cross the floor plate and project into the contralateral VLF (Fig. $5 B, B^{\prime}$ ). In Robo1/2 mutant embryos, most axons still enter the contralateral VLF, but, in $38 \%$ of the injection sites, post-crossing axons were observed in the contralateral gray matter (Fig. 5C, $C^{\prime}$ ). This was only rarely $(7 \%)$ observed in wild-type mice or Robol and Robo2 single mutants (data not shown). These results indicate that Robol and Robo2 collaborate to prevent post-crossing axons from entering the contralateral gray matter. Moreover, these data are consistent with the idea that misguided post-crossing axons account for at least some of the ectopic L1 immunoreactivity in Robo1/2 mutants (and potentially Slit1/2/3 mutants).

\section{Discussion}

The floor plate at the spinal cord ventral midline presents an intermediate target for commissural axons as they grow toward their final destinations. Initially, commissural axons are attracted to the floor plate, but they switch off attraction and become repelled after floor plate crossing. Axonal repellents of the Slit family and their cognate receptors of the Robo family contribute to midline repulsion of commissural axons. To understand the contributions of individual Robo family members to midline axon guidance, we analyzed single and combination mutants of Robo1, Robo2, and Robo3. We provide evidence for the existence of an additional Slit receptor and show that Robo1 and Robo 2 collaborate to prevent post-crossing commissural axons from reentering the gray matter. We also demonstrate that Robo1 and Robo2 have specialized functions in sorting axons into distinct positions within the VLF.

\section{Existence of another Slit receptor}

Analysis of mice lacking Robo1 and Robo2 by TAG-1 immunohistochemistry and DiI injection (Fig. 1) revealed several differences from the Slit1/2/3 triple mutant. First, the defasciculation of pre-crossing commissural axons that is observed in mice lacking all Slits is not observed in Robo1/2 double mutants. Second, TAG-1 is expressed on commissural axons in the most medial part of the VLF in Robol/2 mutants but not in Slit1/2/3 mutants. Third, although there is an increased number of growth cones in the floor plate of mice lacking both Robo1 and Robo2, suggesting axonal stalling, this phenotype is less severe than in mice lacking all Slits, and recrossing of axons, as seen in Slit1/2/3 mutants, is never observed in Robo1/2 mutants. These results indicate that some Slit functions are independent of Robol and Robo2. Robo3.2 can act as a classical Slit receptor and might account for some of these differences. Consistent with this idea, small interfering RNA-mediated knockdown of Robo3.2 in a Robo1/2 mutant increases the number of growth cones in the floor plate, suggesting stalling, and causes some axons to recross the midline (Chen et al., 2008). However, these experiments did not address the other two phenotypic differences between Slit1/2/3 mutants and Robo1/2 mutants. Unlike Robo1 and Robo2, which are expressed at low levels on pre-crossing axons (Long et al., 2004), Robo3.2 appears to be expressed exclusively on the post-crossing portion of commissural axons (Chen et al., 2008). This tends to argue against Robo3.2 mediating the effect of Slits on fasciculation of commissural axons before crossing and for the existence of another receptor, although we cannot exclude that levels of Robo3.2 too low to be detected can mediate this effect.

Robo3.1 is necessary for midline crossing of commissural axons, and Robo3-deficient commissural axons are prematurely responsive to Slits (Sabatier et al., 2004; Chen et al., 2008). Analysis of midline crossing in Robo1/2/3 mutant embryos (Fig. 2) showed that a significant portion of axons cross the midline, suggesting that Robo3 allows midline crossing of some axons by suppressing midline repulsion mediated by Robo1 and Robo2. Because there is more midline crossing in Robol/2/3 mutants than in Robo1/3 mutants but no rescue of crossing in Robo2/3 mutants, these data indicate that some commissural axons in the Robo3 mutant are prevented from crossing because they express Robo1, whereas others are prevented from crossing as a result of expression of both Robo1 and Robo2. However, there is still a significant portion of commissural axons that do not cross the midline in mice lacking Robo1, Robo2, and Robo3. A similar result has been reported for axons of precerebellar neurons of the inferior olive. These axons normally cross the hindbrain floor plate, are prevented from crossing in the Robo3 mutant, and are not rescued in Robo1/2/3 mutants (Di Meglio et al., 2008). Hence, there must be additional mechanisms by which Robo3 allows midline crossing. One possibility is that Robo3 suppresses Slit repulsion through an additional receptor, such as the one postulated above. Alternatively, Robo3 might suppress midline repulsion by an independent ligand/receptor pair, e.g., Semaphorins/Plexins. Finally, 
Robo3 could also potentially function as a receptor for a midline attractant, as discussed previously (Sabatier et al., 2004). Additional studies will be required to distinguish these possibilities.

Robol can silence axonal attraction to Netrin by physically interacting with deleted in colorectal cancer (DCC) during Slitmediated receptor activation (Stein and Tessier-Lavigne, 2001). If there is an additional Slit receptor, it will be interesting to see whether this receptor can suppress axonal responses to Netrin or whether Slit-mediated repulsion and DCC silencing can be separated.

\section{A novel role for Robol and Robo2 in preventing post-crossing axons from entering the gray matter}

In mice lacking all Slits, elevated L1 and Robol immunoreactivity is observed in association with pre-crossing commissural axons (Long et al., 2004). Similarly, we observed increased L1 expression in the Robol/2 double mutant (Fig. 3). To determine whether the elevated L1 staining is a consequence of strongly L1-positive post-crossing commissural axons entering the gray matter and associating with contralateral pre-crossing axons, we traced commissural axons in the Robo1/2 mutant by DiI injection in transverse sections and found that there are indeed postcrossing axons entering the gray matter in this mutant (Fig. 5). This phenotype was not observed in Robol or Robo2 single mutants (data not shown), indicating that Robol and Robo2 collaborate to prevent post-crossing axons from reentering the spinal cord gray matter. All three Slits are expressed in the motor column of the developing spinal cord (Itoh et al., 1998; Brose et al., 1999; Li et al., 1999), suggesting that motor-neuron-derived Slits might confine post-crossing commissural axons to the VLF through Robol and Robo2.

\section{Robo-dependent sorting of axons into different parts of the VLF}

Ectopic expression of a dominant-negative Robol in chick commissural neurons leads to a medial shift of these axons after floor plate crossing (Reeber et al., 2008), indicating that interfering with Robo function can disrupt mediolateral sorting of commissural axons. However, these results did not address which Robo family member(s) is required for correct axonal positioning after midline crossing. We analyzed the post-crossing behavior of commissural axons in Robo1, Robo2, and Robo1/2 mutant embryos and found that axons stay in the VF in mice lacking Robo2, whereas lack of Robo1 causes stalling after floor plate exit and lack of rostral turning in some cases (Fig. 3). Surprisingly, this stalling is rescued in mice lacking both Robo1 and Robo2, indicating that axonal stalling after crossing in Robol mutants is caused by Robo2. These results demonstrate that Robo2 is necessary for axons to leave the immediate vicinity of the floor plate and project into more lateral positions, whereas Robol prevents axons from stalling after crossing. Moreover, these results suggest that the complement of Robos expressed by different subpopulations of commissural axons determines their projection pattern in the contralateral VLF: Robo1/3-positive axons normally project along the floor plate, and Robol/2/3-positive axons project into more lateral positions. Consistent with this idea, Robo1 is expressed throughout the VLF, whereas Robo2 levels appear higher in the LF than in the VF (Long et al., 2004). Slit/ Robo signaling appears to act in concert with Ephrin signaling in mediolateral sorting of commissural axons, because axons are shifted laterally when EphB3/EphB signaling is blocked (Imondi and Kaprielian, 2001; Kadison et al., 2006). Although we did not observe any gross pre-crossing axon guidance defects in the dif- ferent Robo mutants, we cannot exclude the possibility that some of the post-crossing phenotypes described here are indirectly caused by subtle pre-crossing defects and not by a lack of Robo signaling in post-crossing axons.

We found that the function of Robo 2 in directing axons laterally is not restricted to post-crossing axons, because ipsilaterally projecting axons in Robo2/3 mutants fail to leave the vicinity of the floor plate as well (Fig. 4). Consistent with the idea that Robo family members can control mediolateral positioning of diverse axonal populations in the vertebrate spinal cord, longitudinal axon tracts originating from midbrain neurons are shifted medially after genetic deletion of Robo1 and Robo2 in mice (Farmer et al., 2008) or Robo2 in fish (Kastenhuber et al., 2009). These results are reminiscent of Robo-dependent shifts of longitudinal axon tracts in the ventral nerve cord of Drosophila (Rajagopalan et al., 2000; Simpson et al., 2000), suggesting that mediolateral axon tract positioning in the CNS of invertebrates and vertebrates is regulated by evolutionarily conserved mechanisms using Robo family members.

The LF was more dramatically thinned in mice lacking Robo1 and Robo2 than in Robo2 mutant mice, although loss of Robo1 alone did not affect LF thickness (Fig. 3). These data suggest a synergistic effect of the two Robos on the ability of some axons to sort into more lateral positions. It is possible that, in some axonal subpopulations, the combined levels of Robol and Robo 2 contribute to mediolateral positioning. Interestingly, a recent study examining the roles of Robos in lateral tract selection is consistent with this idea, because the overall levels of Robos were the determining factor in some lateral tract positioning decisions in Drosophila (Spitzweck et al., 2010).

\section{References}

Brose K, Bland KS, Wang KH, Arnott D, Henzel W, Goodman CS, TessierLavigne M, Kidd T (1999) Slit proteins bind Robo receptors and have an evolutionarily conserved role in repulsive axon guidance. Cell 96:795-806.

Charron F, Stein E, Jeong J, McMahon AP, Tessier-Lavigne M (2003) The morphogen sonic hedgehog is an axonal chemoattractant that collaborates with netrin-1 in midline axon guidance. Cell 113:11-23.

Chen Z, Gore BB, Long H, Ma L, Tessier-Lavigne M (2008) Alternative splicing of the robo3 axon guidance receptor governs the midline switch from attraction to repulsion. Neuron 58:325-332.

Colamarino SA, Tessier-Lavigne M (1995) The role of the floor plate in axon guidance. Annu Rev Neurosci 18:497-529.

Di Meglio T, Nguyen-Ba-Charvet KT, Tessier-Lavigne M, Sotelo C, Chédotal A (2008) Molecular mechanisms controlling midline crossing by precerebellar neurons. J Neurosci 28:6285-6294.

Farmer WT, Altick AL, Nural HF, Dugan JP, Kidd T, Charron F, Mastick GS (2008) Pioneer longitudinal axons navigate using floor plate and Slit/ Robo signals. Development 135:3643-3653.

Grieshammer U, Le Ma, Plump AS, Wang F, Tessier-Lavigne M, Martin GR (2004) SLIT2-mediated ROBO2 signaling restricts kidney induction to a single site. Dev Cell 6:709-717.

Imondi R, Kaprielian Z (2001) Commissural axon pathfinding on the contralateral side of the floor plate: a role for B-class ephrins in specifying the dorsoventral position of longitudinally projecting commissural axons. Development 128:4859-4871.

Itoh A, Miyabayashi T, Ohno M, Sakano S (1998) Cloning and expressions of three mammalian homologues of Drosophila slit suggest possible roles for Slit in the formation and maintenance of the nervous system. Brain Res Mol Brain Res 62:175-186.

Kadison SR, Mäkinen T, Klein R, Henkemeyer M, Kaprielian Z (2006) EphB receptors and Ephrin-B3 regulate axon guidance at the ventral midline of the embryonic mouse spinal cord. J Neurosci 26:8909-8914.

Kaprielian Z, Runko E, Imondi R (2001) Axon guidance at the midline choice point. Dev Dyn 221:154-181.

Kastenhuber E, Kern U, Bonkowsky JL, Chien CB, Driever W, Schweitzer J (2009) Netrin-DCC, Robo-Slit, and heparan sulfate proteoglycans coordinate lateral positioning of longitudinal dopaminergic diencephalospinal axons. J Neurosci 29:8914-8926. 
Kennedy TE, Serafini T, de la Torre JR, Tessier-Lavigne M (1994) Netrins are diffusible chemotropic factors for commissural axons in the embryonic spinal cord. Cell 78:425-435.

Li HS, Chen JH, Wu W, Fagaly T, Zhou L, Yuan W, Dupuis S, Jiang ZH, Nash W, Gick C, Ornitz DM, Wu JY, Rao Y (1999) Vertebrate slit, a secreted ligand for the transmembrane protein roundabout, is a repellent for olfactory bulb axons. Cell 96:807-818.

Long H, Sabatier C, Ma L, Plump A, Yuan W, Ornitz DM, Tamada A, Murakami F, Goodman CS, Tessier-Lavigne M (2004) Conserved roles for Slit and Robo proteins in midline commissural axon guidance. Neuron 42:213-223.

Ma L, Tessier-Lavigne M (2007) Dual branch-promoting and branchrepelling actions of Slit/Robo signaling on peripheral and central branches of developing sensory axons. J Neurosci 27:6843-6851.

Plump AS, Erskine L, Sabatier C, Brose K, Epstein CJ, Goodman CS, Mason CA, Tessier-Lavigne M (2002) Slit1 and Slit2 cooperate to prevent premature midline crossing of retinal axons in the mouse visual system. Neuron 33:219-232.

Rajagopalan S, Vivancos V, Nicolas E, Dickson BJ (2000) Selecting a longitudinal pathway: Robo receptors specify the lateral position of axons in the Drosophila CNS. Cell 103:1033-1045.

Reeber SL, Sakai N, Nakada Y, Dumas J, Dobrenis K, Johnson JE, Kaprielian Z (2008) Manipulating Robo expression in vivo perturbs commissural axon pathfinding in the chick spinal cord. J Neurosci 28:8698-8708.

Sabatier C, Plump AS, Le Ma, Brose K, Tamada A, Murakami F, Lee EY, Tessier-Lavigne M (2004) The divergent Robo family protein rig-1/
Robo3 is a negative regulator of slit responsiveness required for midline crossing by commissural axons. Cell 117:157-169.

Shirasaki R, Katsumata R, Murakami F (1998) Change in chemoattractant responsiveness of developing axons at an intermediate target. Science 279:105-107.

Simpson JH, Bland KS, Fetter RD, Goodman CS (2000) Short-range and long-range guidance by Slit and its Robo receptors: a combinatorial code of Robo receptors controls lateral position. Cell 103:1019-1032.

Spitzweck B, Brankatschk M, Dickson BJ (2010) Distinct protein domains and expression patterns confer divergent axon guidance functions for Drosophila Robo receptors. Cell 140:409-420.

Stein E, Tessier-Lavigne M (2001) Hierarchical organization of guidance receptors: silencing of netrin attraction by slit through a Robo/DCC receptor complex. Science 291:1928-1938.

Tessier-Lavigne M, Goodman CS (1996) The molecular biology of axon guidance. Science 274:1123-1133.

Yuan SS, Cox LA, Dasika GK, Lee EY (1999) Cloning and functional studies of a novel gene aberrantly expressed in RB-deficient embryos. Dev Biol 207:62-75.

Yuan W, Rao Y, Babiuk RP, Greer JJ, Wu JY, Ornitz DM (2003) A genetic model for a central (septum transversum) congenital diaphragmatic hernia in mice lacking Slit3. Proc Natl Acad Sci U S A 100:5217-5222.

Zou Y, Stoeckli E, Chen H, Tessier-Lavigne M (2000) Squeezing axons out of the gray matter: a role for slit and semaphorin proteins from midline and ventral spinal cord. Cell 102:363-375. 